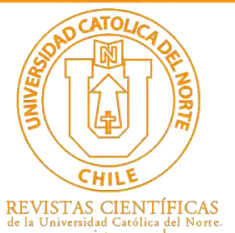

\title{
Rainbow and strong rainbow connection number for some families of graphs
}

\author{
Yaqoub Ahmed Khan ${ }^{1}$ [0 orcid.org/0000-0001-9033-0916 \\ Muhammad Naeem² (1) orcid.org/0000-0002-0919-8207 \\ Muhammad Kamran Siddiqui ${ }^{3}$ (1) orcid.org/0000-0002-2607-4847 \\ Mohammad Reza Farahani ${ }^{4}$ (D) orcid.org/0000-0003-2185-5967 \\ ${ }^{1}$ Government College University, Dept. of Mathematics, Lahore, Pakistan. \\ yaqoubahmedkhan@gmail.com \\ ${ }^{2}$ Institute of Southern Punjab, Dept.of Mathematics and Statistics, Multan, Pakistan. \\ naeempkn@gmail.com \\ ${ }^{3}$ COMSATS University Islamabad, Dept. of Mathematics, Lahore, Pakistan. \\ kamransiddiqui75@gmail.com \\ ${ }^{4}$ Iran University of Science and Technology, Dept. of Applied Mathematics, Tehran, Iran. \\ —mr_farahani@mathdep.iust.ac.ir \\ Received: February 2020 | Accepted: May 2020
}

\section{Abstract:}

Let $G$ be a nontrivial connected graph. Then $G$ is called a rainbow connected graph if there exists a coloring $c: E(G) \rightarrow\{1,2, \ldots, k\}, k \in N$, of the edges of $G$, such that there is a $u-v$ rainbow path between every two vertices of $G$, where a path $P$ in $G$ is a rainbow path if no two edges of $P$ are colored the same. The minimum $k$ for which there exists such a k-edge coloring is the rainbow connection number $\operatorname{rc}(G)$ of $G$. If for every pair $u, v$ of distinct vertices, $G$ contains a rainbow $u-v$ geodesic, then $G$ is called strong rainbow connected. The minimum $k$ for which $G$ is strong rainbow-connected is called the strong rainbow connection number $\operatorname{src}(G)$ of $G$.

The exact $r c$ and src of the rotationally symmetric graphs are determined.

Keywords: Edge coloring; Rainbow coloring; Strong rainbow coloring.

$\operatorname{MSC}(2020): 05 C 15,05 C 38,05 C 40$.

\section{Cite this article as (IEEE citation style):}

Y. A. Khan, M. Naeem, M. K. Siddiqui, and M. Reza Farahani, "Rainbow and strong rainbow connection number for some families of graphs", Proyecciones (Antofagasta, On line), vol. 39, no. 4, pp. 737-747, Aug. 2020, doi: 10.22199/issn.0717-6279-2020-040046.

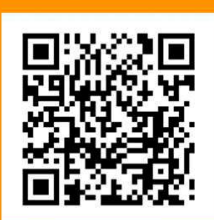

Article copyright: @ 2020 Yaqoub Ahmed Khan, Muhammad Naeem, Muhammad Kamran Siddiqui and Mohammad Reza Farahani. This is an open access article distributed under the terms of the Creative Commons License, which permits unrestricted use and distribution provided the original author and source are credited. 


\section{Introduction}

G. Chartrand in [5] introduce the notion of a rainbow coloring of a graph. He gave some basic and characteristic theorems about the $r c$ and src numbers of graphs. Since then the rainbow connection and strong rainbow connection numbers is studied by many mathematicians.

Let $G$ be a nontrivial connected graph on which an edge - coloring $c: E(G) \rightarrow\{1,2, \ldots, n\}, n \in \mathbf{N}$, is defined, where adjacent edges may be colored the same. A path in $G$ is called a rainbow path if no two edges of this are colored the same. An edge color graph $G$ is called rainbow connected if there is a rainbow path between every two vertices of $G$. An edge-coloring under which $G$ is rainbow connected is called a rainbow coloring. The rainbow connection number of a connected graph $G$, denoted by $\operatorname{rc}(G)$, is the smallest number of colors that are needed in order to make $G$ rainbow connected. A rainbow coloring using $\operatorname{rc}(G)$ colors is called a minimum rainbow coloring.

For any two vertices $u$ and $v$ of $G$, a rainbow $u-v$ geodesic in $G$ is a rainbow $u-v$ path of length $d(u, v)$, where $d(u, v)$ is the distance between $u$ and $v$ in $G$. A graph $G$ is strong rainbow connected if there exists a rainbow $u-v$ geodesic for any two vertices $u$ and $v$ in $G$. In this case, the coloring $c$ is called a strong rainbow coloring of $G$. The strong rainbow connection number of a connected graph $G$, denoted by $\operatorname{src}(G)$, is the smallest number of colors that are neededto make $G$ a strong rainbow connected graph. A strong rainbow coloring of $G$ using $\operatorname{src}(G)$ colors is calleda minimum strong rainbow coloring of $G$. Note that this number is also called the rainbow diameternumber in [4].

If we assign distinct colors to each the edge of a connected graph then both coloring exists namely a rainbow coloring and a strong rainbow coloring, therefore every connected graph is rainbow-connected and strongly rainbow-connected with respect to some coloring of the edges of $G$. So, naturally the rainbow connection numbers $r c(G)$ and $\operatorname{src}(G)$ are defined for every connected graph $G$ and moreover $r c(G) \leq \operatorname{src}(G)$. Also if $G$ is a nontrivial connected graph of size $m$ whose diameter (the largest distance between two vertices of $G$ ) is $\operatorname{diam}(G)$, then

$$
\operatorname{diam}(G) \leq r c(G) \leq \operatorname{src}(G) \leq m .
$$

In this paper we have determined the exact value for $r c$ and $s r c$ number of the graphs of möbius ladder $M_{2 n}$, antiprism $A_{n}$, convex polytope $R_{n}$ and convex polytope $Q_{n}$. 
For further reading in this regards the readers are referred to [15] and [13]. Moreover, Dewi Estetikasari and Syafrizal Sy in [6] determined the exact values of rainbow connection for some corona graph. For more details on different kind of labeling we refer to the articles $[1,5,7,8,9,10,11,12,14,16,17,18]$.

\section{Möbius ladders}

The Möbius ladders $M_{2 n}$ is a cubic circulant graph with an even number of vertices, formed from an $n$-cycle by adding vedges (called rungs) connecting opposite pairs of vertices in the cycle, we denote the outer vertices as $u_{i}$, $1 \leq i \leq n$ and inner vertices as $v_{i}, 1 \leq i \leq n$. It is so-named because (with the exception of $\left.M_{6}=K_{3,3}\right) M_{2 n}$ has exactly $n$ number of 4-cycles which are linked together by their shared edges to form a topological Möbius strip. Möbius ladder can also be viewed as a prism with one twisted edge. Two different views of Möbius ladders $M_{16}$ have been shown in Fig 2.1 Möbius ladders have many applications in chemistry, chemical stereography, electronics and computer science.
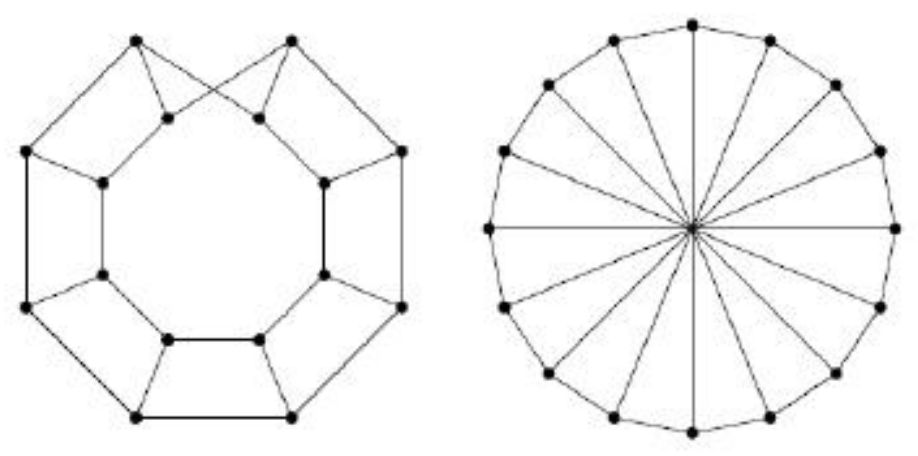

2.1. Two views of Möbius ladders $M_{2 n}$

Möbius ladder has $2 n$ vertices and $3 n$ edges for all $n \geq 2$ with diameter of $M_{2 n}$ is equal to $\lceil n / 2\rceil$ then its easy to see from (1.1) that

$$
\operatorname{src}\left(M_{2 n}\right) \geq r c\left(M_{2 n}\right) \geq\lceil n / 2\rceil
$$


Theorem 1. The rainbow connection number and the strong rainbow connection number of Möbius ladder $M_{2 n}$ is $\lceil n / 2\rceil$ for $n \geq 2$.

Proof. It is easy to see that $M_{2 n}$ contain a $2 n$-cycle, $C_{2 n}: u_{1}, u_{2}, \ldots, u_{n}$, $v_{1}, v_{2}, \ldots, v_{n}, u_{1}$. We denote the edges of $M_{2 n}$ as: $e_{1 i}=u_{i} u_{i+1}, e_{2 i}=v_{i} v_{i+1}$ for $1 \leq i \leq n-1, e_{3 i}=u_{i} v_{i}$ for $1 \leq i \leq n, e_{4 n}=u_{n} v_{1}$ and $e_{5 n}=v_{n} u_{1}$.

Let us take $\lceil n / 2\rceil=k$ for our convenience. To show that $\operatorname{src}\left(M_{2 n}\right) \leq k$ we define the edge coloring $c: E\left(M_{2 n}\right) \rightarrow\{1,2,3 \ldots, k\}$ as follows:

$$
c\left(e_{j i}\right)= \begin{cases}i, & \text { for } j=1,2 \text { and } 1 \leq i \leq k \\ i-k, & \text { for } j=1,2 \text { and } k<i \leq n-1 \\ i, & \text { for } j=3 \text { and } 1 \leq i \leq k \\ i-k, & \text { for } j=1,2 \text { and } k<i \leq n \\ \lfloor n / 2\rfloor, & \text { for } j=3,4 \text { and } i=n .\end{cases}
$$

From $c$ we get $\operatorname{src}\left(M_{2 n}\right) \leq k=\lceil n / 2\rceil$. Thus from (1.1) and (2.1) we obtain $\operatorname{src}\left(M_{2 n}\right)=r c\left(M_{2 n}\right)=\lceil n / 2\rceil$. To understand the above coloring see the Fig 2.2 for $M_{16}$.

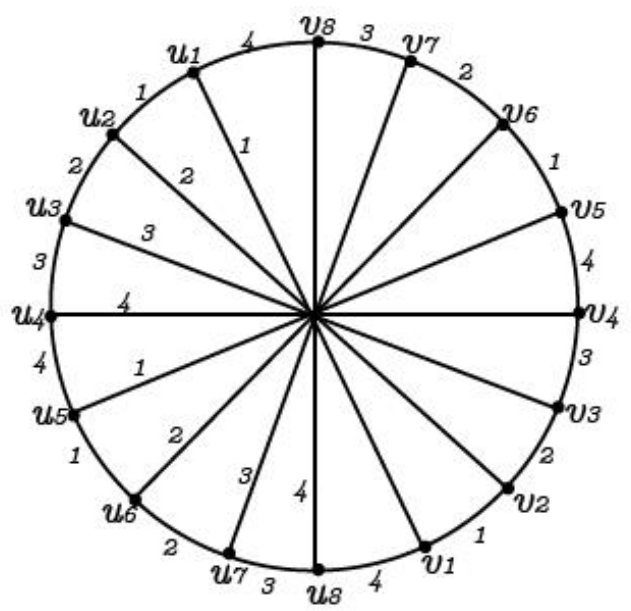

2.2. Rainbow and strong rainbow coloring of $M_{16}$

\section{The graph of an antiprism}

The antiprism $A_{n}, n \geq 3$, is a 4 -regular graph and, for $n=3$, it is the octahedron. This graph has $2 n$ vertices and $4 n$ edges for $n \geq 3, A_{n}$ consists 
of an outer $n$-cycle $v_{1} v_{2} \ldots v_{n}$, an inner $n$-cycle $u_{1} u_{2} \ldots u_{n}$, and a set of $n$ spokes $u_{i} v_{i}$ and $u_{i+1} v_{i}$ for $i=1,2, \ldots n$ with indices taken modulo $n$ see (Fig. 3.1).

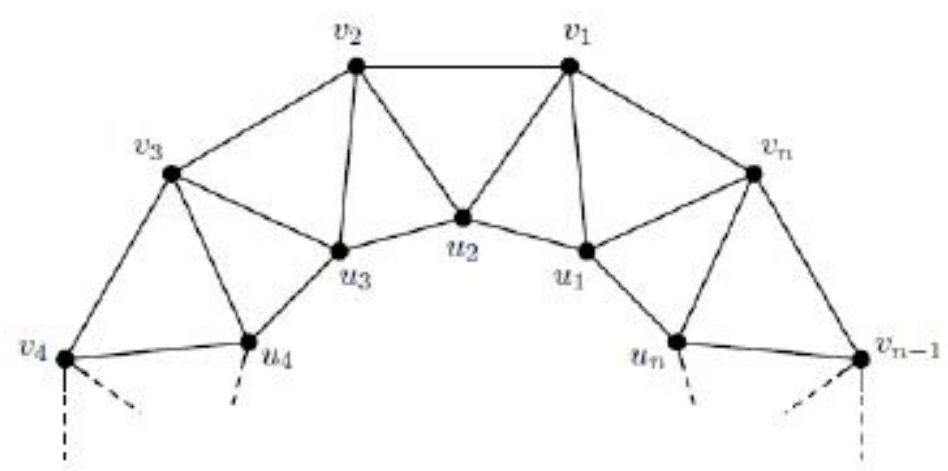

3.1. The graph of antiprism $A_{n}$

The diameter of $A_{n}$ for $n \geq 3$ is $\lceil n / 2\rceil$. So by (1.1) we obtain

$$
\operatorname{src}\left(A_{n}\right) \geq \operatorname{rc}\left(A_{n}\right) \geq\lceil n / 2\rceil
$$

Theorem 2. The rainbow connection number and the strong rainbow connection number of Antiprism $A_{n}$, for $n \geq 3$ is $\lceil n / 2\rceil$.

Proof. We denote the edges of $A_{n}$ as: $e_{1 i}=v_{i} v_{i+1}, e_{2 i}=u_{i} u_{i+1}$ for $1 \leq i \leq n, e_{3 i}=u_{i} v_{i}$ for $1 \leq i \leq n, e_{4 n}=u_{i+1} v_{i}$ for $1 \leq i \leq n$. Let us take $\lceil n / 2\rceil=k$ for our convenience. From (3.1) we already know that $\operatorname{src}\left(A_{n}\right) \geq k$. To show that $\operatorname{src}\left(A_{n}\right) \leq k$ we define the edge coloring $c: E\left(A_{n}\right) \rightarrow\{1,2,3 \ldots, k\}$ as follows:

$$
c\left(e_{j i}\right)= \begin{cases}i, & \text { for } 1 \leq j \leq 4 \text { and } 1 \leq i \leq k \\ i-k, & \text { for } 1 \leq j \leq 4 \text { and } k<i \leq n\end{cases}
$$

This coloring $c$ on $E\left(A_{n}\right)$ give us a rainbow $u-v$ geodesic for any two vertices $u$ and $v$ in $A_{n}$. From $c$ we get $\operatorname{src}\left(A_{n}\right) \leq k=\lceil n / 2\rceil$. Thus from (1.1) and (3.1) we obtain $\operatorname{src}\left(A_{n}\right)=r c\left(A_{n}\right)=\lceil n / 2\rceil$. To understand the 
above coloring see the Fig 3.2 for $A_{8}$.

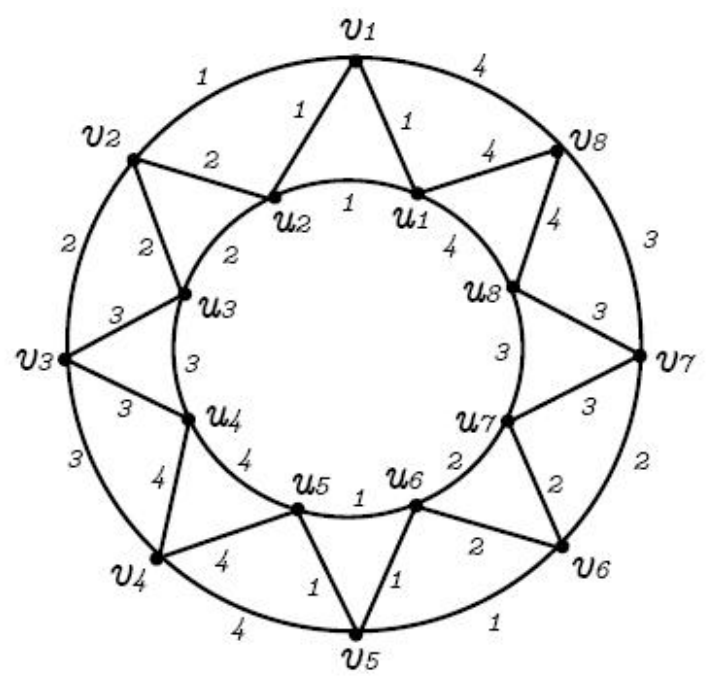

\section{The graph of convex polytope $R_{n}$}

For $n \geq 3$, by $R_{n}$, we denote the graph of convex polytope defined in $[2,3,10,11,12,14]$ which is obtained as a combination of the graph of a prism and the graph of an antiprism (Fig. 4.1). The graph $R_{n}$ has $3 n$ vertices and $6 n$ edges for $n \geq 3$. The $\operatorname{diam}\left(R_{n}\right)=\lceil n / 2\rceil+1$. By using (1.1)

$$
\operatorname{src}\left(R_{n}\right) \geq r c\left(R_{n}\right) \geq\lceil n / 2\rceil+1
$$

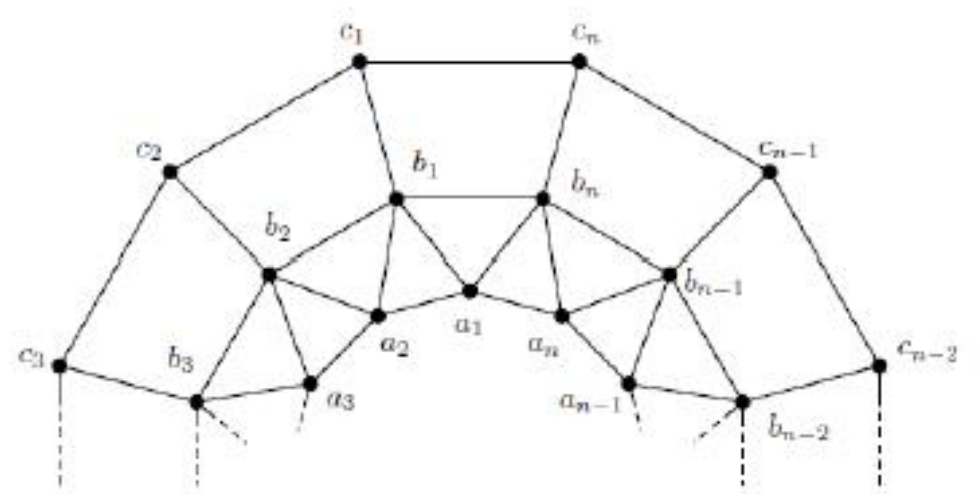

4.1. The graph of convex polytop $R_{n}$ 
Theorem 3. For $n \geq 3$, the rainbow connection number and strong connection number of convex polytop $R_{n}$ is $\lceil n / 2\rceil+1$.

Proof. The graph $R_{n}$ contains three $n$-cycles, $A_{n}: a_{1}, a_{2}, a_{3}, \ldots, a_{n}, a_{1}$, $B_{n}: b_{1}, b_{2}, b_{3}, \ldots, b_{n}, b_{1}$ and $C_{n}: c_{1}, c_{2}, c_{3}, \ldots, c_{n}, c_{1}$, with edges denoted as $e_{1 i}=a_{i} a_{i+1}, e_{2 i}=b_{i} b_{i+1}, e_{3 i}=c_{i} c_{i+1}$ respectively for $1 \leq i \leq n$. The cycle $A_{n}$ is connected to $B_{n}$ by $n$ spoke edges denoted as $e_{4 i}=a_{i} b_{i}$ and $e_{5 i}=a_{i+1} b_{i}$ for $1 \leq i \leq n$. The cycle $B_{n}$ is connected to $C_{n}$ by the edges denoted as $e_{6 i}=b_{i} c_{i}$ for $1 \leq i \leq n$.

Let $\lceil n / 2\rceil+1=k+1$, then from (4.1) we have $\operatorname{src}\left(R_{n}\right) \geq k+1$. To show that $\operatorname{src}\left(R_{n}\right) \leq k$ we define the edge coloring $c: E\left(R_{n}\right) \rightarrow\{1,2,3, \ldots, k+1\}$ as follows:

$$
c\left(e_{j i}\right) \begin{cases}i, & \text { for } 1 \leq j \leq 5 \text { and } 1 \leq i \leq k \\ i-k, & \text { for } 1 \leq j \leq 5 \text { and } k<i \leq n \\ k+1, & \text { for } j=6 \text { and } 1 \leq i \leq n\end{cases}
$$

The coloring function $c$ gave us a coloring for $R_{n}$ in which there exists a rainbow path as well as a rainbow geodesic path for each pair of vertices of $R_{n}$. This shows that $\operatorname{src}\left(R_{n}\right) \leq k+1$. Thus the result follows from (1.1) and the formulas $c\left(e_{j i}\right)$ in above equation.

\section{The graph of convex polytope $Q_{n}$}

In this section, we shall investigate the $r c\left(Q_{n}\right)$ and $\operatorname{src}\left(Q_{n}\right)$ of the graph of convex polytope $Q_{n}$ for $n \geq 3$ defined in consisting of 3 - sided faces, 4 - sided faces, $5-$ sided faces and $n$-sided faces (Fig. 5.1). The graph of $Q_{n}$ have $4 n$ vertices and $7 n$ edges, for $n \geq 3$ with $\operatorname{diam}\left(Q_{n}\right)=\lceil n / 2\rceil+2$. So from (1.1) it follows 


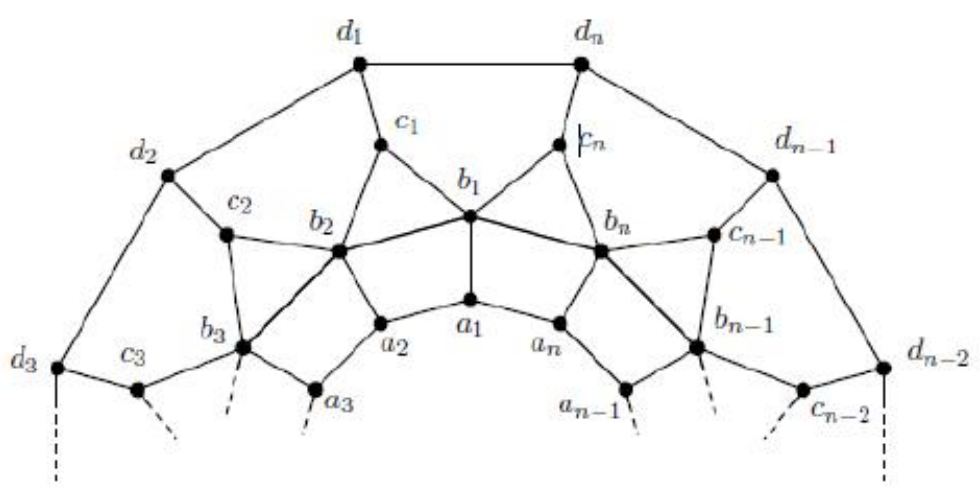

5.1 The graph of convex polytop $Q_{n}$

$$
\operatorname{src}\left(Q_{n}\right) \geq r c\left(Q_{n}\right) \geq\lceil n / 2\rceil+2
$$

Theorem 4. For $n \geq 3$, the rainbow connection number of convex polytop $Q_{n}$ is $\lceil n / 2\rceil+2$.

Proof. The graph of $Q_{n}$ contains three $n$-cycles $A_{n}: a_{1}, a_{2}, a_{3}, \ldots, a_{n}, a_{n+1}=$ $a_{1}, B_{n}=b_{1}, b_{2}, b_{3}, \ldots, b_{n}, b_{n+1}=b_{1}$ and $D_{n}=d_{1}, d_{2}, d_{3}, \ldots, d_{n}, d_{n+1}=d_{1}$ having the edges denoted as $e_{1 i}=a_{i} a_{i+1}, e_{2 i}=b_{i} b_{i+1}$, and $e_{3 i}=d_{i} d_{i+1}$ respectively for $1 \leq i \leq n$. The cycle $A_{n}$ is connected to $B_{n}$ by the edges denoted by $e_{4 i}=a_{i} b_{i}$ for $1 \leq i \leq n$. Wedenotes as $e_{5 i}=b_{i} c_{i}$ and $e_{6 i}=b_{i+1} c_{i}$, and the edges defined $c_{i} d_{i}$ are denoted as $e_{7 i}$ for $1 \leq i \leq n$.

For $n=3$, consider 4-coloring $c: E\left(Q_{n}\right) \rightarrow\{1,2,3,4\}$, which gives us a rainbow coloring of $Q_{3}$, defined as $c\left(e_{j i}\right)=i$ for $j=1,2,3,4,5,7$, $1 \leq i \leq 3$ and $c\left(e_{6 i}\right)=41 \leq i \leq 3$. Since the $\operatorname{diam}\left(Q_{3}\right)=4$, so it results that $r c\left(Q_{3}\right)=4$.

Now for $n \geq 4$, first we let $\lceil n / 2\rceil+2=k+2$ then we define $(k+2)-$ 
rainbow coloring $c: E\left(Q_{n}\right) \rightarrow\{1,2,3, \ldots, k+2\}$ as follows:

$$
c\left(e_{j i}\right)= \begin{cases}i, & \text { for } 1 \leq j \leq 6, j \neq 4 \text { and } 1 \leq i \leq k \\ i-k, & \text { for } 1 \leq j \leq 6 j \neq 4 \text { and } k<i \leq n \\ k+1, & \text { for } j=4 \text { and } 1 \leq i \leq k \\ k+1, & \text { for } j=7 \text { and } k<i \leq n \\ k+2, & \text { for } j=7 \text { and } 1 \leq i \leq k \\ k+2, & \text { for } j=4 \text { and } k<i \leq n\end{cases}
$$

Under this coloring there is a rainbow path between each pair of vertices of $Q_{n}$, for $n \geq 4$. So

$\operatorname{rc}\left(\mathrm{Q}_{n}\right) \leq\lceil n / 2\rceil+2$ Finally by relating with equation (5.1), $r c\left(Q_{n}\right)=$ $\lceil n / 2\rceil+2$

\section{References}

[1] M. Alaeiyan and M. R. Farahani, "The 1-2-3-edge labeling and vertex colors", International journal of applied mathematics and machine learning, vol. 4, no. 2, pp. 119-133, 2016, doi: 10.18642/ijamml_7100121608

[2] F. Asif, Z. Zahid, S. Zafar, M. R. Farahani, and W. Gao, "On topological properties of some convex polytopes by using line operator on their subdivisions", Hacettepe journal of mathematics and statistics, vol. 49, no. 3, pp. 136-146, Jan. 2019, doi: 10.15672/HJMS.2019.671

[3] M. Baĉa, "On magic labellings of convex polytopes", Annals of discrete mathematics, pp. 13-16, 1992, doi: 10.1016/S0167-5060(08)70599-5

[4] S. Chakraborty, E. Fischer, A. Matsliah, and R. Yuster, "Hardness and algorithms for rainbow connectivity", Leibniz international proceedings in informatics, pp. 243-254, 2009, doi: 10.4230/LIPIcs.STACS.2009.1811

[5] G. Chartrand, G. L. Johns, K. A. McKeon, and P. Zhang, "Rainbow connection in graphs", Mathematica bohemica, vol. 133, no. 1, pp. 85-98, 2008. [On line]. Available: https://bit.ly/2AJRJwz 
[6] D. Estetikasari and S. Sy, "On the rainbow connection for some corona graphs", Applied mathematical sciences, vol. 7, no. 100, pp. 4975-4980, 2013, doi: 10.12988/ams.2013.37410

[7] M. R. Farahani, "On The 1-2-3-edge weighting and vertex coloring of complete graph", International journal on computational science \& applications, vol. 3, no. 3, pp. 19-23, Jun. 2013, doi: 10.5121/ijcsa.2013.3302

[8] M. R. Farahani, "A new vertex-coloring edge-weighting of complete graphs", Journal of applied mathematics \& informatics, vol. 32, no. 1_2, pp. 1-6, Jan. 2014, doi: 10.14317/jami.2014.001

[9] M. R. Farahani and S. H. Hosseini "The 1-2-3-edge labeling and vertex coloring of complete graph Kn with a modified algorithm", Algebras, Groups and Geometries, vol. 31, no. 2, pp. 183-199, 2014. [On line]. Available: https://bit.ly/3fth3pa

[10] Z. Foruzanfar, F. Asif, Z. Zahid, S. Zafar, and M.R. Farahani, " $\mathrm{ABC}_{4}$ and $\mathrm{GA}_{5}$ indices of line graph of subdivisions of convex polytopes", International journal of pure and applied mathematics, vol. 117, no. 4, pp. 645-653, 2017. [On line]. Available: https://bit.ly/3hC8sTi

[11] Z. Foruzanfar, F. Asif, Z. Zahid, S. Zafar, and M. R. Farahani, " $\mathrm{ABC}_{4}$ and $\mathrm{GA}_{5}$ indices of para-line graph of some convex polytope", Statistics, optimization \& information computing, vol. 7, no. 1, pp. 192-197, Jan. 2019, doi: 10.19139/soic.v7i1.346

[12] M. N. Husin, F. Asif, Z. Zahid, S. Zafar, and M. R. Farahani, "Fourth atombond connectivity index and fifth arithmetic-geometric index of convex polytopes by using line operator", Advances and applications in discrete mathematics, vol. 19, no. 4, pp. 491-500, Oct. 2018, doi: 10.17654/DM019040491

[13] X. Li, Y. Shi, and Y. Sun, "Rainbow connections of graphs: a survey", Graphs and combinatorics, vol. 29, no. 1, pp. 1-38, Oct. 2012, doi: 10.1007/s00373-012-1243-2

[14] R. Sohail, A. Rehman, M. Sh. R. Chowdhury, M. Imran, and M. R. Farahani, "Topological indices of some convex polytopes", International journal of pure and applied mathematics, vol. 119, no. 3, pp. 451-460, 2018. [On line]. Available: https://bit.ly/2N69Waf 
[15] S. Sy, R. Wijaya, and Surahmat, "Rainbow connection numbers of some graphs", Applied mathematical sciences, vol. 8, no. 94, pp. 4693-4696, 2014, doi: 10.12988/ams.2014.46398

[16] B. Yang, M. Rashid, S. Ahmad, M. Nadeem, and M. Siddiqui, "Cycle super magic labeling of planar graphs", International journal of applied mathematics, vol. 32, no. 6, pp. 945-957, 2019, doi: 10.12732/ijam.v32i6.4

[17] H. Yang, M. A. Rashid, S. Ahmad, M. K. Siddiqui, and M. F. Hanif, "Cycle super magic labeling of pumpkin, octagonal and hexagonal graphs", Journal of discrete mathematical sciences and cryptography, vol. 22, no. 7, pp. 1165-1176, Dec. 2019, doi: 10.1080/09720529.2019.1698800

[18] L. Yan, Y. Li, X. Zhang, M. Saqlain, S. Zafar, and M. R. Farahani, "3-total edge product cordial labeling of some new classes of graphs", Journal of information and optimization sciences, vol. 39, no. 3, pp. 705-724, Apr. 2018, doi: 10.1080/02522667.2017.1417727 